\title{
Modelación matemática para el diseño mecánico de una plataforma autocargable.
}

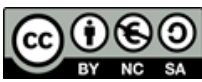

\section{Mathematical modeling for the mechanical design of a self-loading platform.}

Luis Patricio Tierra Pérez. ${ }^{1}$, Sócrates Miguel Aquino Arroba ${ }^{2}$, Edwin Rodolfo Pozo Safla $^{3}$ \& Eduardo Santiago Cazar Rivera ${ }^{4}$

Recibido: 28-04-2019 / Revisado: 24-05-2019 /Aceptado: 28-06-2019/ Publicado: 25-07-2019

\section{Abstract. \\ DOI: https://doi.org/10.33262/cienciadigital.v3i3.2.727}

Summary- This document describes the design steps of a self-loading platform, the system for the lifting of the load is a mechanism crank with slide.

(i) Similar models are reviewed by consulting the bibliography and development, the loading conditions are analyzed, the most suitable model is weighted and selected with a QFD matrix, the user is related to the options for the design.

(ii) The methods used in the research are by direct observation when analyzing the existing models in the market, exploratory, empirical-analytical because a model is selected and then analyzed with computer programs such as Matlab and Solidworks. a proactive investigation because it chooses an effective solution to the problem;

(iii) Analyzes have been carried out by means of analytical and software calculations, arriving to consider that the maximum load is in the pivots of the system with the

1 Escuela Superior Politécnica de Chimborazo, Facultad de Recursos Naturales, Macas, Ecuador, patricio.tierra@espoch.edu.ec.

2 Superior Politécnica de Chimborazo, Facultad de Mecánica. Riobamba, Ecuador, miguel. aquino@espoch.edu.ec.

3 Escuela Superior Politécnica de Chimborazo, Facultad de Mecánica. Riobamba, Ecuador, edwin.pozo@espoch.edu.ec.

4 Escuela Superior Politécnica de Chimborazo, Facultad de Recursos Naturales, Macas, Ecuador, Eduardo.cazar@espoch.edu.ec. 
loaded platform is $1.2464 \times 10^{\wedge} 5 \mathrm{~N}, 124641$ Newtons $=12709.84$ Kilogram-force, this allows the design of critical accessories.

(iv) With the dynamic analysis it is concluded that when the hydraulic cylinder is tilted more force is needed to raise the load, the proposed model is functional and easily constructed and maintained.

Keywords. Self-loading platform. Matlab, Solidworks, dynamic analysis, hydraulic cylinder.

\section{Resumen.}

Resumen - El presente documento describe los pasos de diseño de una plataforma auto cargable, el sistema para el levantamiento de la carga es un mecanismo tipo biela manivela con corredera.

(i) Se revisan modelos similares consultando la bibliografía y desarrollo, se analizan las condiciones de carga, se pondera y selecciona el modelo más adecuado con una matriz QFD, se relaciona el usuario con las opciones para el diseño.

(ii) Los métodos emlpleados en la investigación son por observación directa cuando se analizan los modelos existentes en el mercado, exploratorio, empíricoanalítico debido a que se selecciona un modelo y luego se analiza con los programas computacionales como el Matlab y SolidWorks, es una investigación propositiva porque elige una la solución efectiva del problema.;

(iii) Se ha realizado los análisis por medio de cálculos analíticos y de software, llegando a considerar que la carga máxima es en los pivotes del sistema con la plataforma cargada es de $1.2464 \times 10^{\wedge} 5 \mathrm{~N}, 124641$ Newtons $=12709.84$ Kilogramo-fuerza, esto permite el diseño de los accesorios críticos.

(iv) Con el análisis dinámico se concluye que cuando el cilindro hidráulico esta inclinado se necesita más fuerza para elevar la carga, el modelo planteado es funcional de fácil construcción y mantenimiento.

Palabras Claves - Plataforma auto-cargable. Matlab, Solidworks, análisis dinámico, cilindro hidráulico. 
El presente documento describe los pasos de diseño de una plataforma auto cargable, el sistema para el levantamiento de la carga es un mecanismo tipo biela manivela con corredera.

Se revisan modelos similares consultando la bibliografía y desarrollo, se analizan las condiciones de carga, se pondera y selecciona el modelo más adecuado con una matriz QFD, se relaciona el usuario con las opciones para el diseño.

Los métodos emlpleados en la investigación son por observación directa cuando se analizan los modelos existentes en el mercado, exploratorio, empírico-analítico debido a que se selecciona un modelo y luego se analiza con los programas computacionales como el Matlab y SolidWorks, es una investigación propositiva porque elige una la solución efectiva del problema.;

Se ha realizado los análisis por medio de cálculos analíticos y de software, llegando a considerar que la carga máxima es en los pivotes del sistema con la plataforma cargada es de $1.2464 \times 10^{\wedge} 5 \mathrm{~N}, 124641$ Newtons $=12709.84$ Kilogramo-fuerza, esto permite el diseño de los accesorios críticos.

Con el análisis dinámico se concluye que cuando el cilindro hidráulico esta inclinado se necesita más fuerza para elevar la carga, el modelo planteado es funcional de fácil construcción y mantenimiento.

\section{Introducción.}

Plataforma auto-cargable; es un equipo usado para el transporte de automotores, maquinaria o equipos de un lugar de origen a su destino, sin que este tenga contacto alguno con la carretera, su construcción depende del diseño y de su necesidad de carga, todas ellas ligadas a un equipo móvil como son los camiones, utiliza el principio de plano inclinado, rampa o pendiente para levantar una carga.

\section{Selección de alternativas.}

Para escoger la mejor alternativa se toman criterios como facilidad de operación tamaño, tiempo de operación, costos de construcción, seguridad, mantenimiento, aceptación en el mercado, tipo de automotor a transportarse. Analizando de cada alternativa las ventajas y desventajas se aplica un modelo matemático. 


\section{Método de la mejor alternativa.}

La mejor alternativa se ha seleccionado por método del valor técnico ponderado que se basa en un conjunto de criterios valorados que suelen tener distinta importancia en el proceso de elección, para lo que es necesario jerarquizar los mismos y asígnale un peso en función a su posición en la evaluación jerárquica.

Para cada alternativa se valora su bondad según el criterio asignándole una puntuación "g" en la escala de 1-10 (gas=10) procediendo seguidamente a determinar el valor técnico ponderado.

Capacidad de carga. De acuerdo a la necesidad del mercado, el primordial requerimiento es la transportación de los montacargas, del análisis de la tabla 3 se determina que la carga máxima se produce en el transporte de los dos montacargas con un peso $7830 \mathrm{~kg}$ y en transporte del tractor con un peso de $6590 \mathrm{Kg}$, de acuerdo al Ministerio de Transporte y Obras Publicas tipifica que para el tipo de camión 2DB (ANEXO A), el peso bruto admisible para circular por las carreteras es $17000 \mathrm{~kg}$, el peso del sistema y carga bruta que va hacer acoplado la plataforma auto-cargable no debe exceder lo recomendado, como también no exceder los $11500 \mathrm{Kg}$ que es la capacidad recomendado por los fabricantes para el chasis camión

Dimensiones. Las dimensiones lo rigen el Ministerio de Transporte y Obras Públicas tomando en cuenta estas dimensiones y las dimensiones máximas que satisfaga el transporte de la tabla 3 tenemos.

Las medidas utilizadas para el diseño, que permiten suplir el transporte en su totalidad de la máxima carga

El sistema oleo hidráulico se usa por su eficiencia y ventaja mecánica, se encuentra relacionado con la estabilidad de la plataforma, la elevación, el deslizamiento, y el arrastre de los automotores o equipos por medio del malacate. 
Se consideró las necesidades para el transporte de la maquinaria, montacargas, automotores. Para el diseño del proyecto considera lo siguiente:

- $\quad$ Capacidad de carga.

- Dimensiones.

- $\quad$ Confiabilidad y seguridad.

- $\quad$ Ambiente de trabajo.

- $\quad$ Vida útil.

- Materiales y construcción.

- Mantenimiento y montaje.

Materiales y Métodos

Se determinan los requerimientos de diseño, que parte de la necesidad de transportar equipos y autos, en donde se hace una selección de los diferentes modelos y pesos, la matriz QFD permite priorizar los requerimientos de diseño con las necesidades del usuario.

En la segunda parte se considera los diferentes modelos existentes en el mercado de equipos capaces transportar y el estudio de su posible construcción.

En la tercera parte corresponde al uso del programa Solidworks para cumplir con los requerimientos de diseño. Haciendo por medio de este programa el análisis funcional. Con la ayuda del software Matlab se desarrolla el análisis cinemático del dispositivo que determina las ecuaciones de posición, velocidad y aceleraciones. Para luego pasar al análisis dinámico del modelo propuesto utilizando el método de trabajos virtuales para obtener las ecuaciones. El software Solidworks, se obtienen datos.

Componentes importantes del rehabilitador tales como: Masas, centros de misa, momentos de inercia. Finalmente utiliza el software Solidwork Simualtion para realizar los análisis de la estructura con teoría de esfuerzos (Von Misses) se determina el factor de seguridad. 
Finalmente, un diseño digital en Solidworks.

\section{Resultado y Discusión}

Para el análisis del proyecto se considera las cargas que soportara la plataforma durante su vida útil las principales son.

- $\quad$ Carga viva

- $\quad$ Carga muerta

- $\quad$ Carga de seguridad

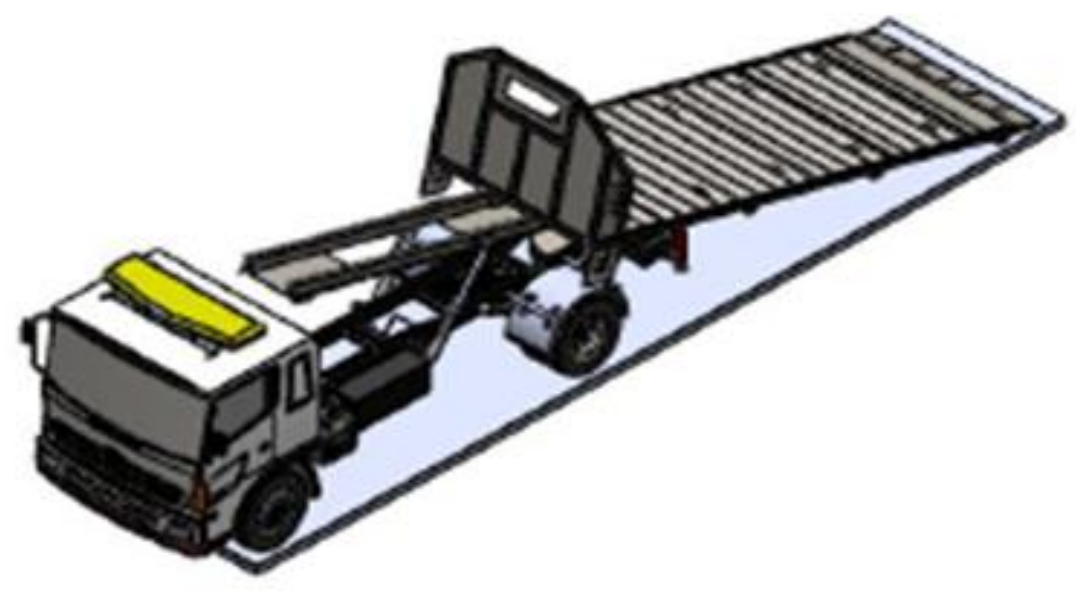

Fig. 1. Modelo seleccionado.

\section{Resultados de la matriz QFD}

De acuerdo al análisis de la matriz QFD mostrados en la fig. 2 los parámetros más relevantes considerados para el diseño son:

- $\quad$ Seguridad para el usuario.

- Usar materiales de bajo costo y existentes en el mercado nacional.

- $\quad$ Diseño del mecanismo adecuado.

- $\quad$ De fácil mantenimiento 


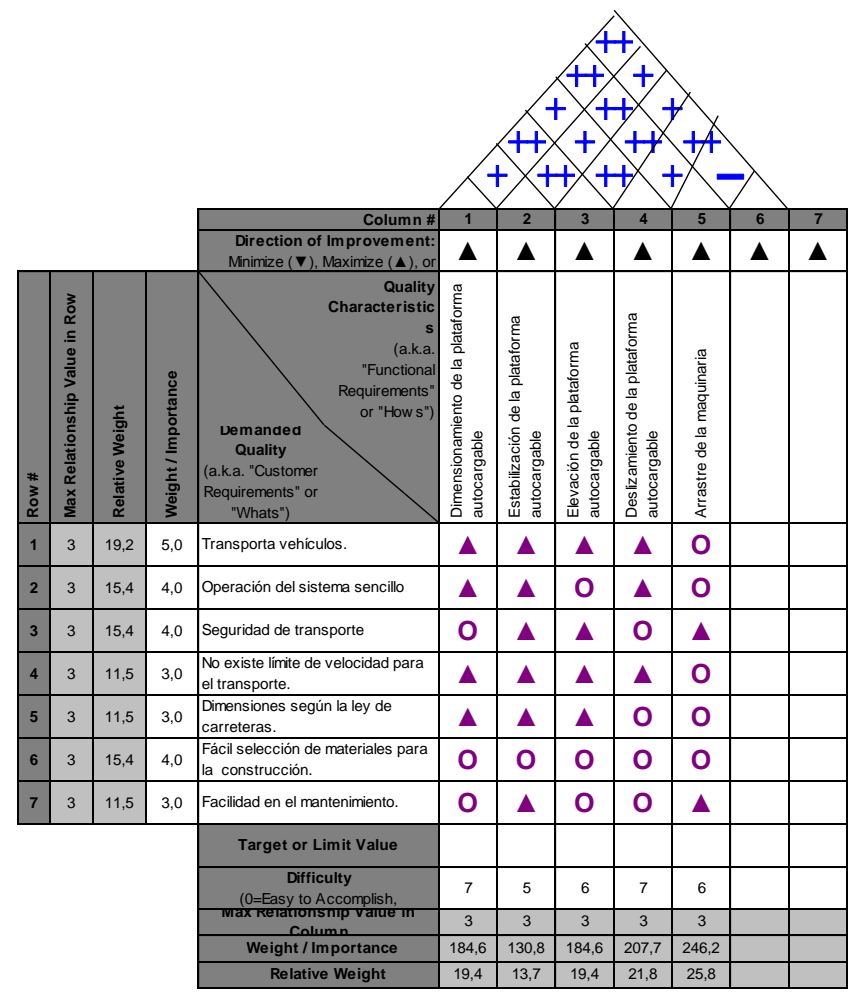

Fig. 2 Matriz QFD

\section{Análisis cinemático}

El sistema de levantamiento de carga es el objeto de estudio y se puede analizar como un sistema de biela manivela de la siguiente manera:

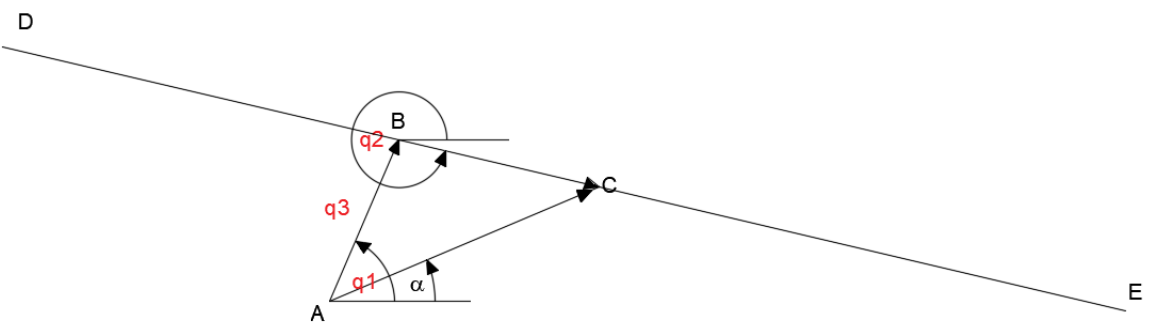




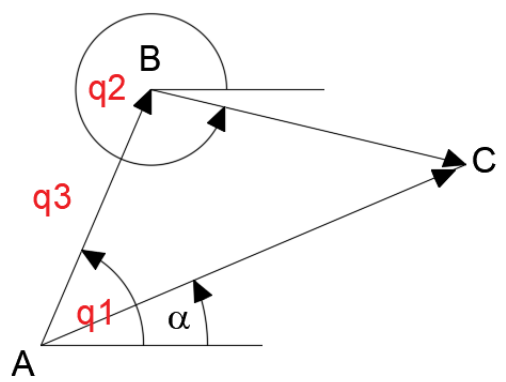

Fig. 3. Gráfico de Lazo analizado ABC

\section{Análisis de posición}

De acuerdo a los diagramas mostrados en la fig. 3 se establecen las ecuaciones para la parte cinemática y las ecuaciones de posición son:

$$
\begin{gathered}
\overrightarrow{r_{A B}}+\overrightarrow{r_{B C}}=\overrightarrow{r_{A C}} \\
q 3 * \cos (q 1)+B C * \cos (q 2)=A C * \cos (\propto) \\
\mathrm{q} 3 * \sin (\mathrm{q} 1)+\mathrm{BC} * \sin (\mathrm{q} 2)=\mathrm{AC} * \sin (\propto)
\end{gathered}
$$

\section{Resultado del análisis posición}
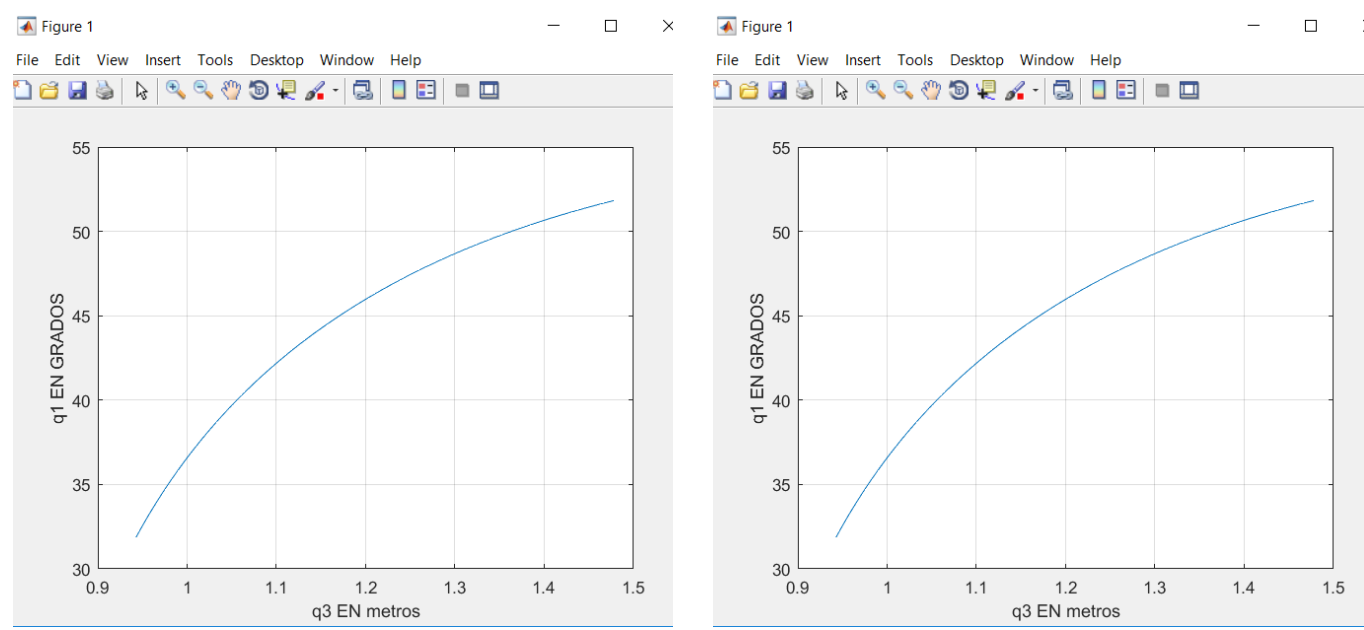

Fig. 4. Graficas de Matlab del análisis de posición 
Estas graficas son adecuadas porque nos permiten observar claramente el desplazamiento del pistón y de la rampa.

\section{Análisis de velocidad}

Derivando las ecuaciones de posición se obtienen las siguientes ecuaciones:

$$
\begin{gathered}
-q 3 * \sin (q 1) * q 1 p-B C * \sin (q 2) * q 2 p+\cos (q 1) * q 3 p=0 \\
q 3 * \cos (q 1) * q 1 p+\sin (q 1) * q 3 p+B C * \cos (q 2) * q 2 p=0
\end{gathered}
$$

\section{Resultado del análisis velocidad}
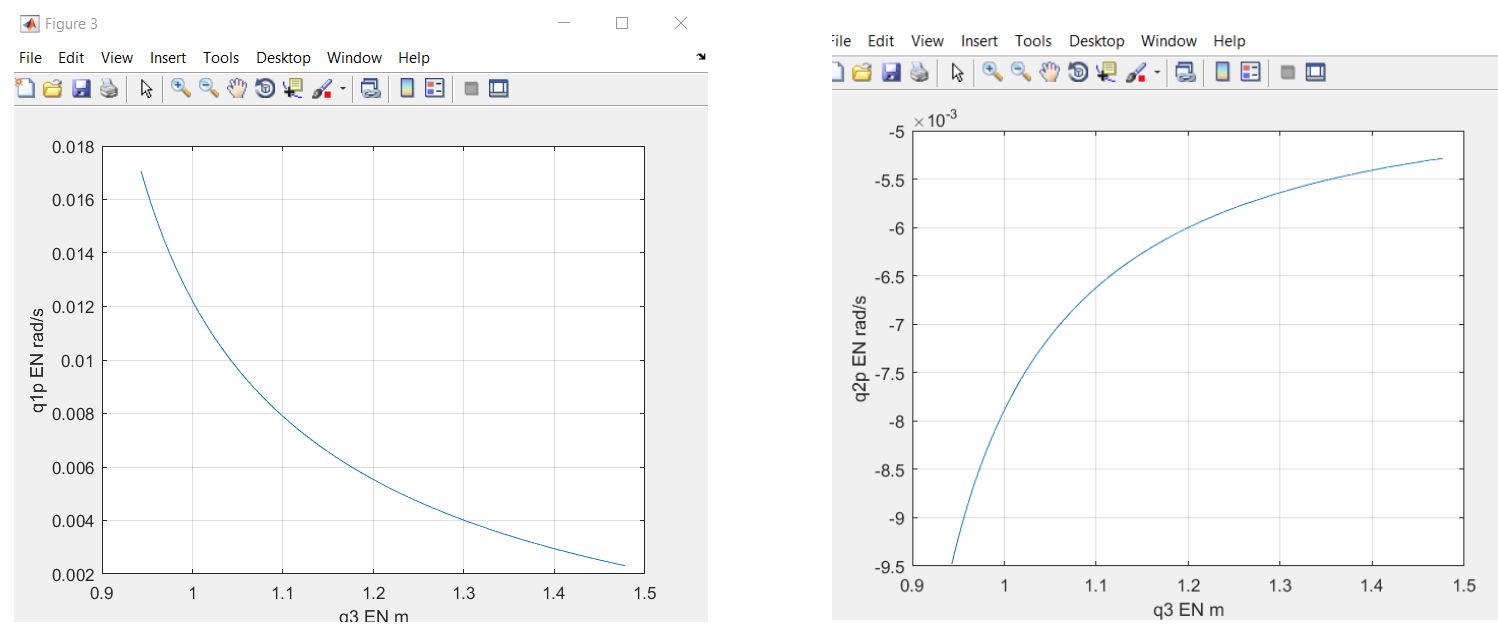

Fig. 5. Graficas de Matlab del análisis de velocidad

\section{Análisis de aceleración}

$$
\begin{gathered}
-q 3 *\left[\sin (q 1) * q 1 p p+q 1 p^{2} * \cos (q 1)\right]-q 3 p * \sin (q 1) * q 1 p+\cos (q 1) * q 3 p p \\
-q 3 p * \sin (q 1) * q 1 p-B C *\left[\sin (q 2) * q 2 p p+q 2 p^{2} * \cos (q 2)\right]=0 \\
q 3 *\left[\cos (q 1) * q 1 p p-q 1 p^{2} * \sin (q 1)\right]+q 3 p * \cos (q 1) * q 1 p+\sin (q 1) * q 3 p p+q 3 p \\
* \cos (q 1) * q 1 p+B C *\left[\cos (q 2) * q 2 p p-q 2 p^{2} * \sin (q 2)\right]=0
\end{gathered}
$$


le Edit View Insert Tools Desktop Window Help

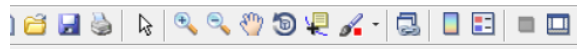
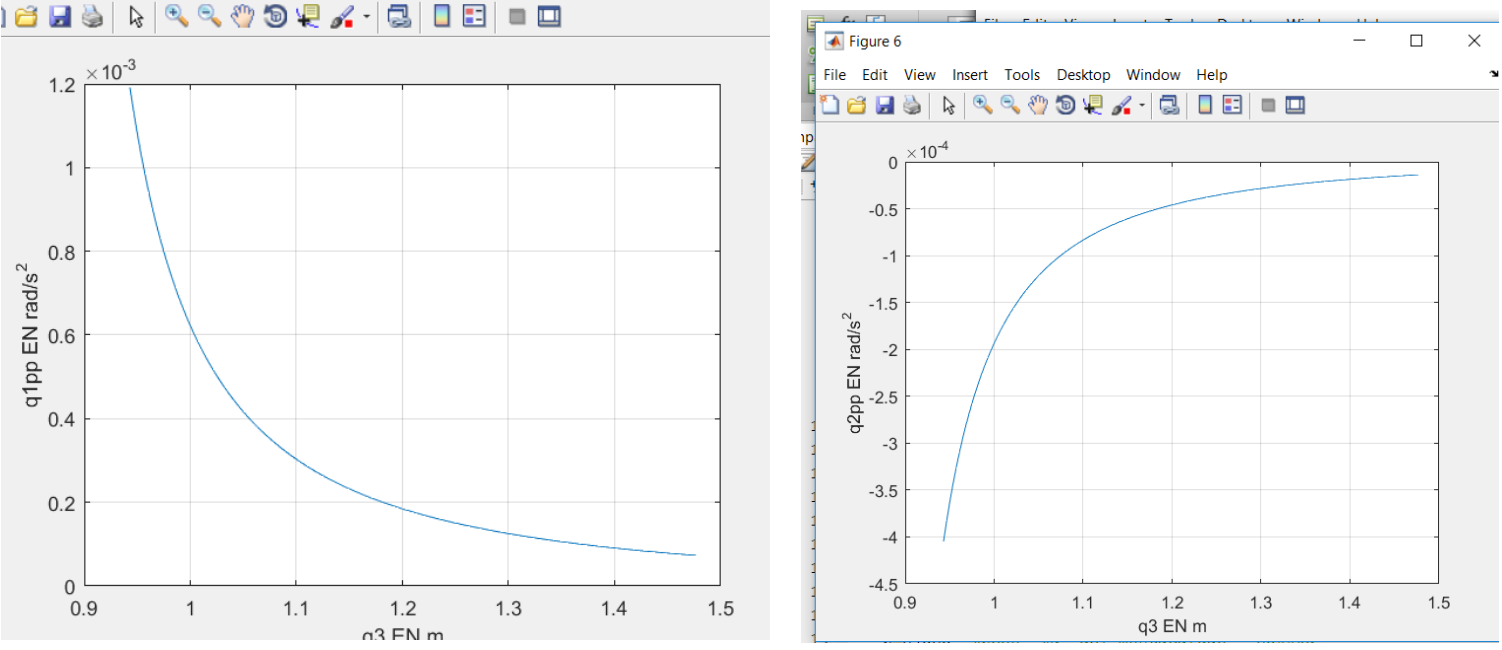

Fig. 6. Graficas de Matlab del análisis de aceleración.

En las gráficas de aceleración se puede determinar un comportamiento típico de un mecanismo biela manivela.

\section{Análisis de posición en los centros de gravedad}

\section{Elemento 1}

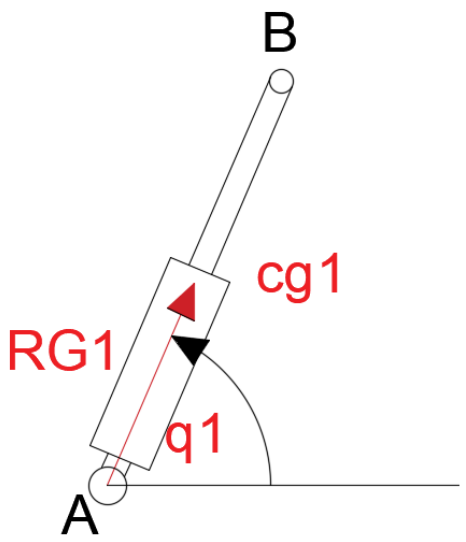

Fig. 7. Análisis de centro de gravedad de cilindro de levantamiento. 


$$
\operatorname{Rg} 1=\left(\begin{array}{c}
\left(\frac{q 3}{2}\right) * \cos (q 1) \\
\left(\frac{q 3}{2}\right) * \cos (q 2) \\
0
\end{array}\right)
$$

\section{Elemento 2}

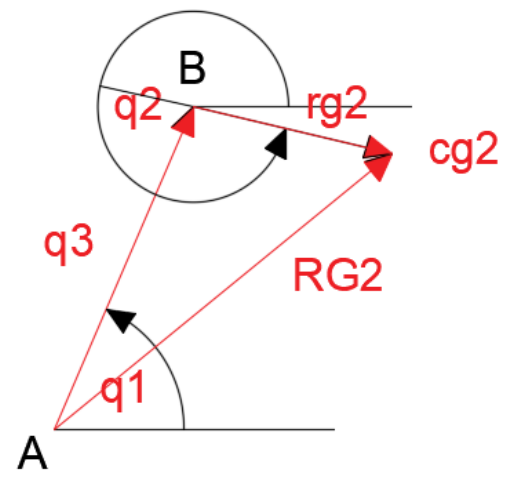

Fig. 7. Diagrama de cuerpo libre de cilindro de levantamiento.

$$
\operatorname{Rg} 2=\left(\begin{array}{c}
q 3 * \cos (q 1)+r g 2 * \cos (q 2) \\
q 3 * \sin (q 1)+r g 2 * \sin (q 2) \\
0
\end{array}\right)
$$

\section{Análisis dinámico de la plataforma}

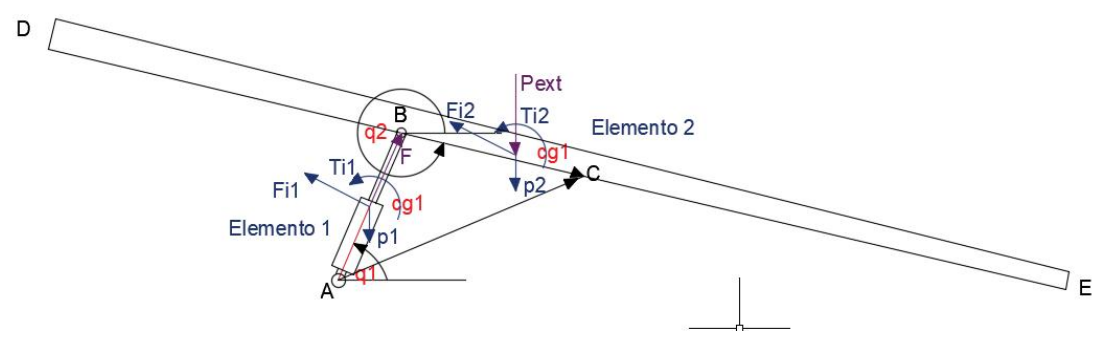

Fig. 10. Esquema para el análisis de dinámico. 
Mediante el software SolidWorks se obtienen las propiedades físicas de los elementos, como masa, momentos de inercia y centros de masa.

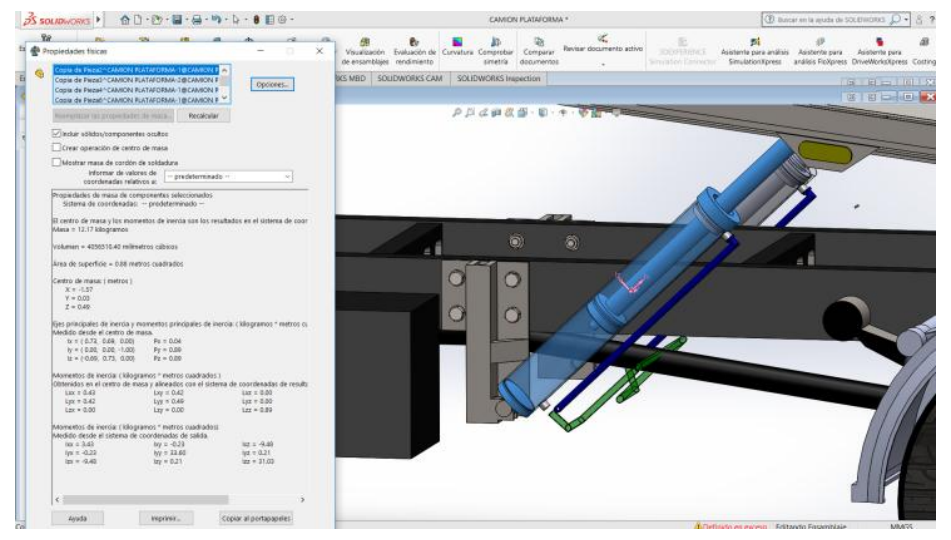

Fig. 10. Calculo de datos geométricos para el elemento 1

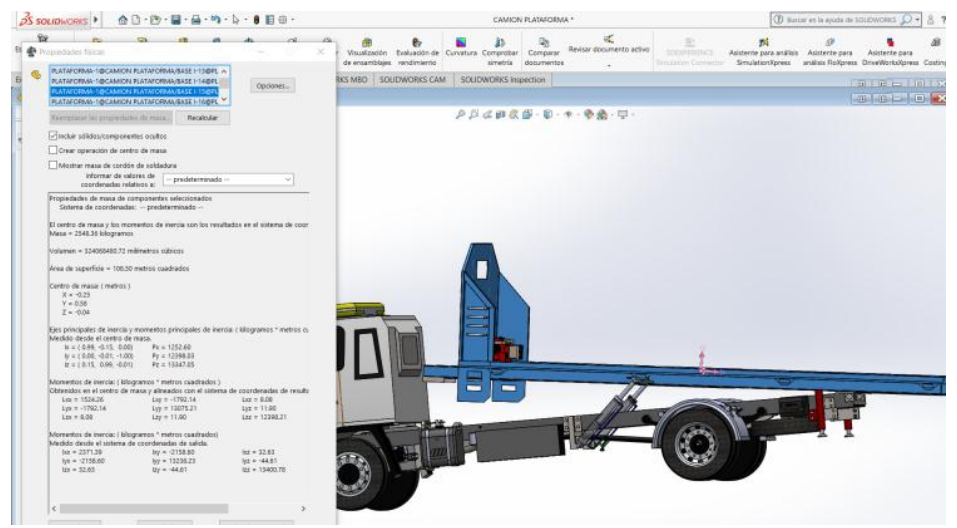

Fig. 10. Calculo de datos geométricos para el elemento 2

\section{Método del trabajo virtual}

El análisis dinámico es realizado con el método de trabajo virtual obteniendo las siguientes ecuaciones:

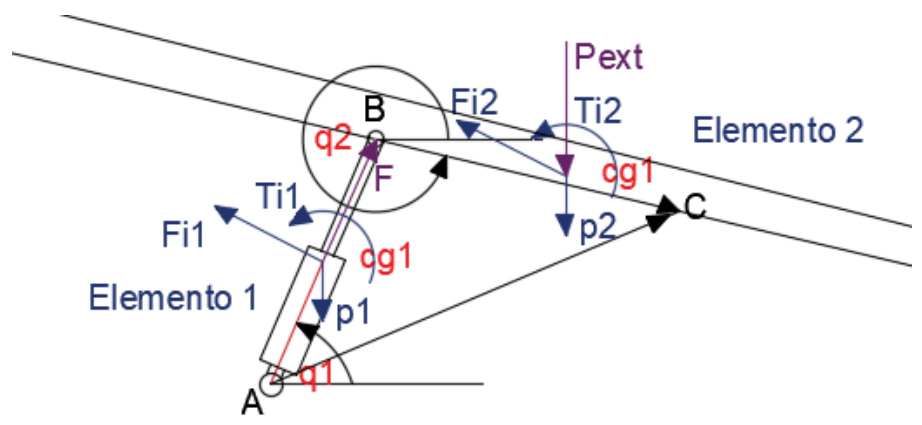

Fig. 11. Esquema para el análisis de dinámico.

\section{Elemento 1}




$$
\begin{gathered}
\overrightarrow{P 1} * \frac{\overrightarrow{\partial R G 1}}{\partial q i}+\overrightarrow{F l 1} * \frac{\overrightarrow{\partial R G 1}}{\partial q i}+\overrightarrow{T \imath 1} * \frac{\overrightarrow{\partial \theta 1}}{\partial q i}+\overrightarrow{F a c t} * \frac{\overrightarrow{\partial R G 1}}{\partial q i} \\
\text { Elemento } 2 \\
\overrightarrow{P 2} * \frac{\overrightarrow{\partial R g 2}}{\partial q i}+\overrightarrow{F l 2} * \frac{\overrightarrow{\partial R g 2}}{\partial q i}+\overrightarrow{T l 2} * \frac{\overrightarrow{\partial \theta 2}}{\partial q i}+\overrightarrow{P e x t} * \frac{\overrightarrow{\partial R g 2}}{\partial q i}=J_{T}^{T} * \gamma
\end{gathered}
$$

\section{Sistema de ecuaciones}

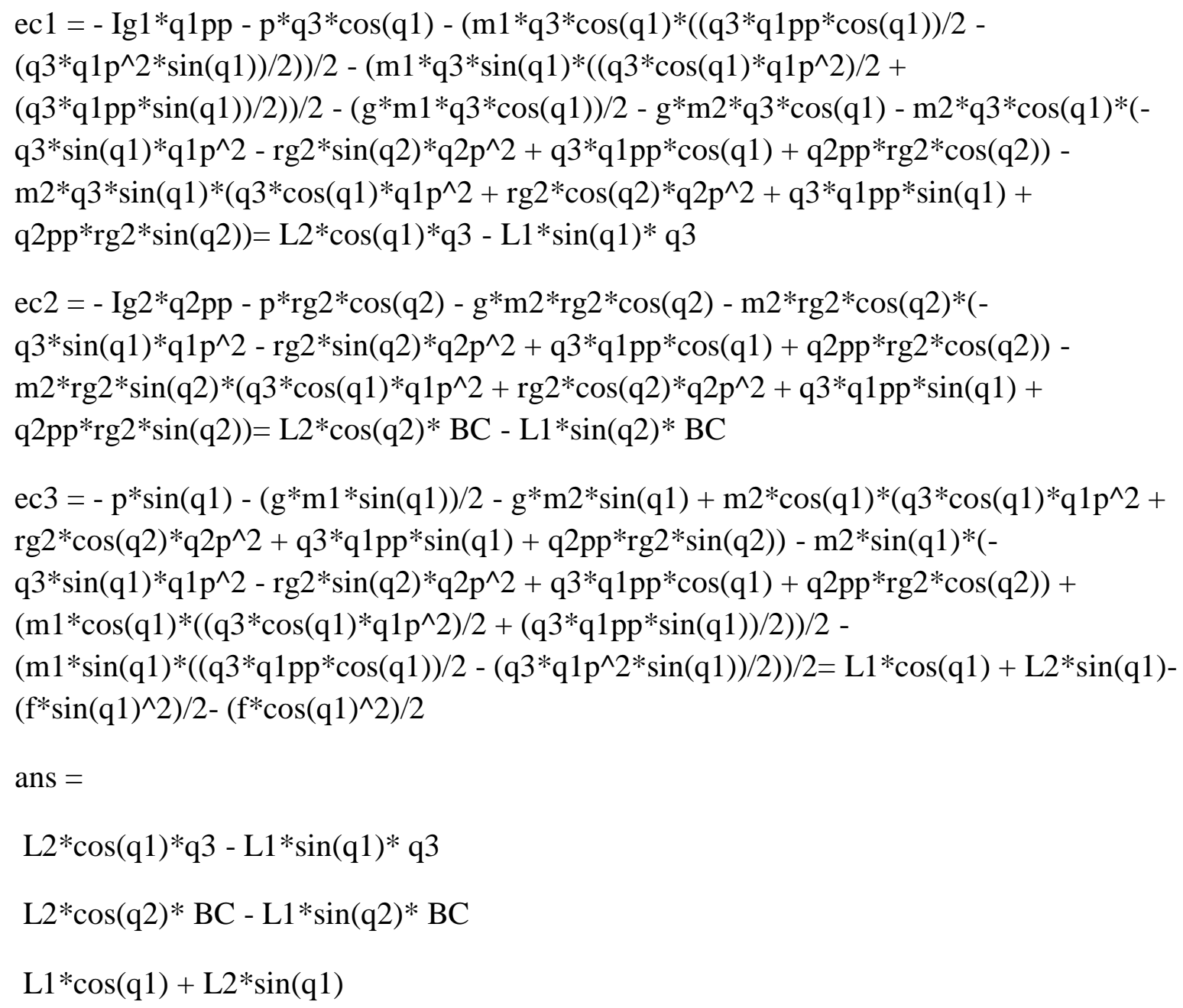

\section{Resultados del análisis dinámico}

El análisis determina que se requiere de una fuerza de $1.2464 \times 10^{\wedge} 5 \mathrm{~N}$ para elevar la carga máxima de diseño. Este resultado nos permite seleccionar o diseñar el cilindro hidráulico para el sistema. Cuando se requiere analizar la resistencia del mecanismo, se la debe hacer 
en esta posición crítica, donde se efectúa la fuerza mayor y se determina la geometría y los materiales.

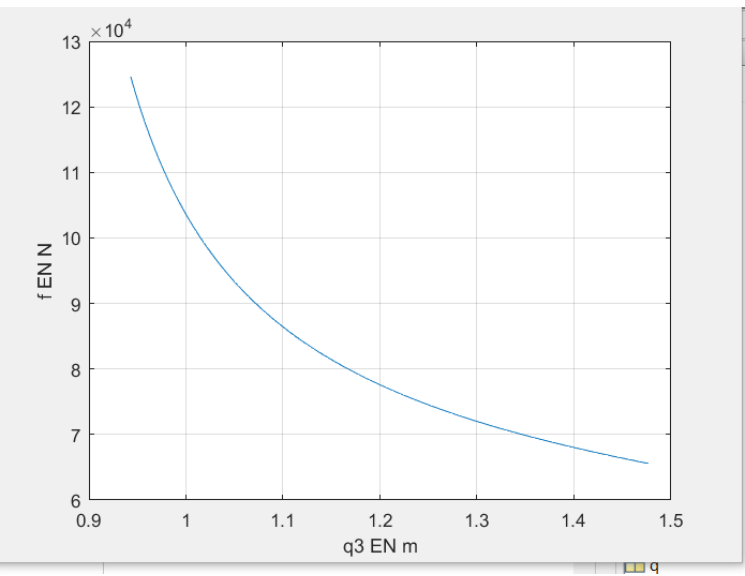

Fig. 12. Resultado del análisis dinámico.

\section{Diseño del soporte y pivotante plataforma.}

Cuando la plataforma se encuentra cargada, Con una reacción máxima en el pivote de 124640 Newtons $=12709.84$ Kilogramo-fuerza determinamos el eje y porta eje que pivoteara al sistema.

$\sqrt{\sigma_{x}+3 \tau_{x y}^{2}}=\frac{S_{y}}{n}$

$\sigma_{x}=$ Esfuerzo axial

$\tau_{x y}=$ Esfuerzo cortante

$S_{y}=$ Resistencia de fluencia

$n=$ coeficiente de seguridad

\section{Determinación del momento flector}

$M=F x L$

$M=12709 \mathrm{Kg} \times 20 \mathrm{~mm}$

$M=213978,4 \mathrm{kgxmm}$ 
$M=$ momento flector

$F=$ Fuerza actuante

$L=$ Longuitud eje

$\sigma_{\text {Flexión }}=\frac{32 M}{\pi x d^{3}}$

$\sigma_{\text {Flexión }}=\frac{32 M}{\pi x d^{3}}$

$\tau=\frac{F}{\frac{\pi}{4} d^{2}}$

$F=$ fuerza actuante

$d=$ diámetro eje

\section{Según Von Mises Hencky}

$\sqrt{\sigma_{x}^{2}+3 \tau_{x y}^{2}}=\frac{S_{y}}{n}$

$\sqrt{\left(\frac{32 M}{\pi x d^{3}}\right)^{2}+3\left(\frac{F}{\frac{\pi}{4} d^{2}}\right)^{2}}=\frac{S_{y}}{n}$

$S_{y}=25,4 \frac{\mathrm{kg}}{\mathrm{mm}^{2}}$

$\sqrt{\left(\frac{32 \times 213978,4 \mathrm{kgxmm}}{\pi d^{3}}\right)^{2}+3\left(\frac{10698,92 \mathrm{~kg}}{\frac{\pi}{4} d^{2}}\right)^{2}}=\frac{25,4 \frac{\mathrm{kg}}{\mathrm{mm}^{2}}}{3}$

$n=$ factor de seguidad 3

$d=68.4 \mathrm{~mm}$

\section{Diseño de los porta ejes}

Al no existir fuerza de corte y estar sometido a compresión calculamos el espesor de las placas que soportan el eje.

$\sigma_{x}=\frac{S_{y}}{n}$ 
$a=\frac{\pi x d x b}{2}$

$a=$ Area media sección circular

$b=$ Longitud placa

$\sigma$ compresión $=\frac{F}{a}$

$\frac{F}{\frac{\pi x d x b}{2}}=\frac{S_{y}}{n}$

$\frac{10698,92}{\frac{\pi x 70 x b}{2}}=\frac{25,4}{3}$

$b=11,5 m m$

Ancho de la placa comercial es de $10 \mathrm{~mm}$ y el más utilizado en la fabricación de los componentes por lo tanto se fusionarán 2 placas.

Comprobación por el método de elementos finitos con Solidworks Simulation:

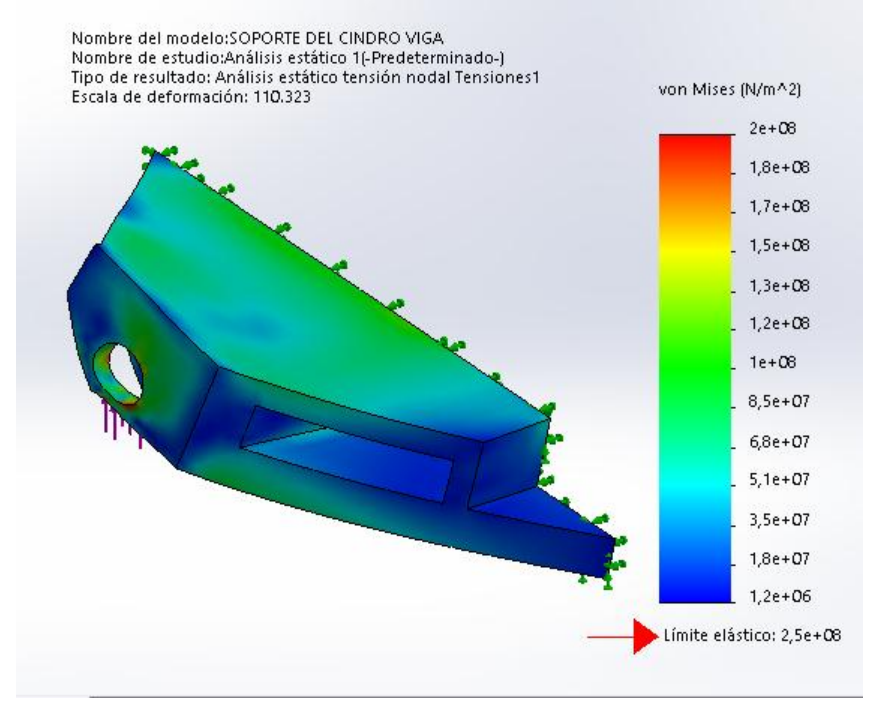

Fig. 13. Resultados del estudio estático con Von Mises.

En la figura anterior se observa un límite elástico de $2 \mathrm{e} 8 \mathrm{~N} / \mathrm{mm}^{2}$ siendo menor que el Sy del material A36 de $2.5 \mathrm{e} 8 \mathrm{~N} / \mathrm{mm}^{2}$. El mallado es uniforme 


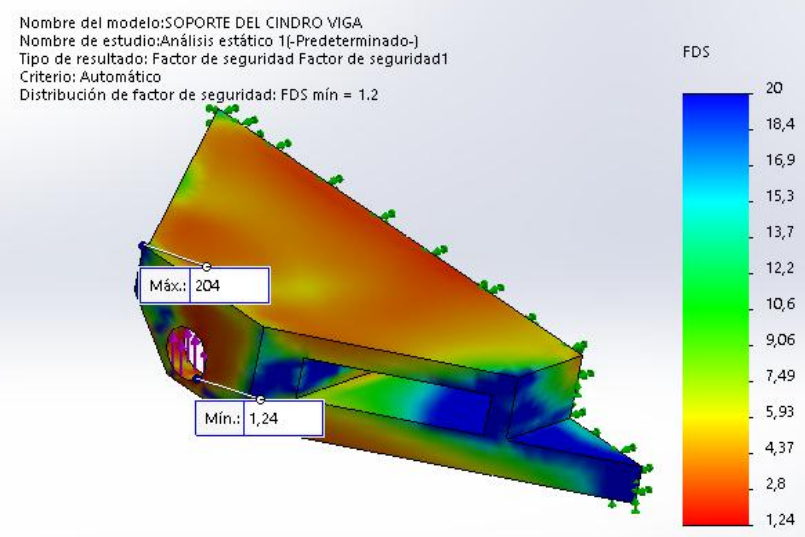

Fig. 14. Resultados del estudio estático factor de seguridad.

La figura anterior muestra un factor de seguridad adecuado de 1.24 , considerando que es una singularidad y no un valor constante en toda la geometría y que esta carga critica seria solo a la máxima capacidad (las cargas de equipos y autos están por debajo de esta exigencia) en un instante de la carga y descarga de la plataforma.

\section{Conclusiones}

- Los métodos matemáticos permiten la realización de los cálculos de una forma rápida, y estos pueden ser corroborados con ensayos reales y con simulaciones que nos permiten determinar las cargas máximas permisibles.

- Se concluye que a mayor condición de carga mayor es el esfuerzo recibido, y este también depende de la posición crítica de la plataforma

- Cuando el cilindro hidráulico está inclinado se necesita más fuerza para elevar la carga, el cilindro dependerá de la presión que va a soportar o la carga que va a levantar.

\section{Bibliografía}

Almandoz, J., Mongelos, B. \& Pallejero, I., 2015. Sistemas neumáticos y oleohidráulico. Murcia: s.n.

Dassault Systémes, S.A., 2018. Solidworks herramienta 3D. Suresnes: s.n.

MTOP, 2015. Capacidad de carga de transporte pesado en Ecuador. Quito: s.n. 
Narro, E., 2017. Aplicación de algunos modelos matemáticos a la toma de decisiones. Mexico DF: s.n.

Ramos, D., Novillo, G. \& Elvis, A., 2015. DISEÑO DE UNA PLATAFORMA AUTOCARGABLE HASTA 8.5 TONELADAS ACOPLADA A UN CHASIS CAMIÓN HINO GDSJLSA. Riobamba: s.n.

Rodriguez, B. \& Marrero, F., 2011. ANUAL DE INVESTIGACION DE OPERACIONES II. Santa Clara: s.n.

Ruiz, A. \& Rojas, F., 2009. DESPLIEGUE DE LA FUNCIÓN CALIDAD (QFD). Madrid: s.n.

Ruiz, C. \& Paredes, F., 2015. ESTUDIO DE PESOS Y DIMENSIONES DE LOS VEHÍCULOS DE CARGA QUE CIRCULAN SOBRE LAS CARRETERAS DE LA RED VIAL ESTATAL ECUATORIANA. Quito: s.n.

Vega, G. \& García, P., 2011. Selección de Alternativas para la Toma de Decisiones. Lima: s.n. 


\section{PARA CITAR EL ARTÍCULO INDEXADO}

J Tierra Pérez, L., Aquino Arroba, S., Pozo Safla, E., \& Cazar Rivera, E. (2019). Modelación matemática para el diseño mecánico de una plataforma autocargable. Ciencia Digital, 3(3.2), 201-220.

https://doi.org/10.33262/cienciadigital.v3i3.2.727

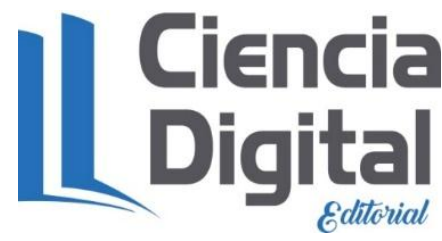

El artículo que se publica es de exclusiva responsabilidad de los autores y no necesariamente reflejan el pensamiento de la Revista Ciencia Digital.

El artículo queda en propiedad de la revista y, por tanto, su publicación parcial y/o total en otro medio tiene que ser autorizado por el director de la Revista Ciencia Digital.
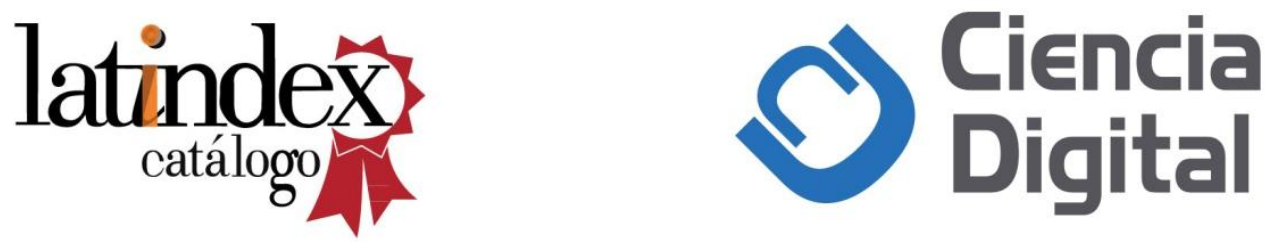